\title{
On the scattering cross section of passive linear arrays
}

\section{Solymar, L.}

\section{Published in:}

I E E E Transactions on Antennas and Propagation

Publication date:

1973

\section{Document Version}

Publisher's PDF, also known as Version of record

Link back to DTU Orbit

Citation (APA):

Solymar, L. (1973). On the scattering cross section of passive linear arrays. I E E E Transactions on Antennas and Propagation, 21(3), 391-392.

\section{General rights}

Copyright and moral rights for the publications made accessible in the public portal are retained by the authors and/or other copyright owners and it is a condition of accessing publications that users recognise and abide by the legal requirements associated with these rights.

- Users may download and print one copy of any publication from the public portal for the purpose of private study or research.

- You may not further distribute the material or use it for any profit-making activity or commercial gain

- You may freely distribute the URL identifying the publication in the public portal

If you believe that this document breaches copyright please contact us providing details, and we will remove access to the work immediately and investigate your claim. 


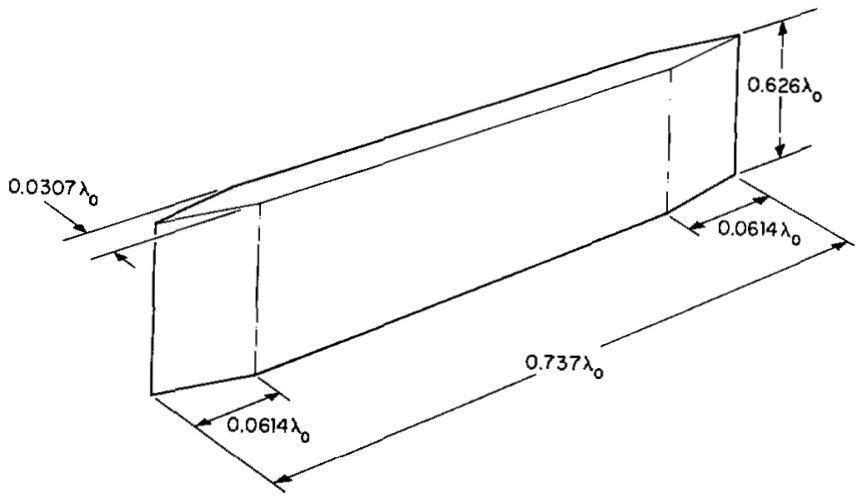

Fig. 4. Geometry of Teflon slab loading for optimum axial ratio condition. Dimensions are not critical.

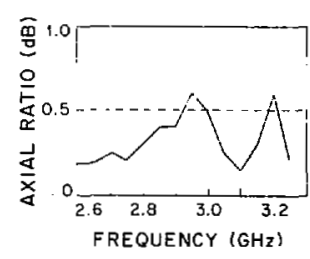

(a)

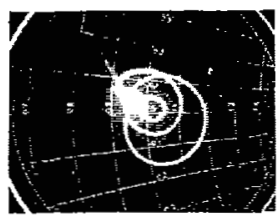

VSWR $\leq 1.2$

(c)

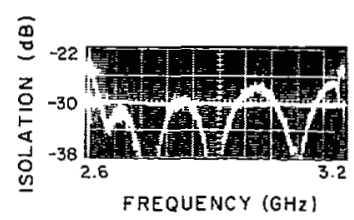

(b)

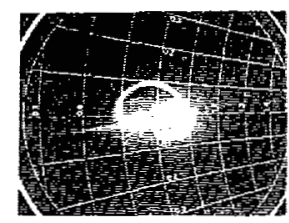

$V S W R \leq 1.2$

(d)
Fig. 5. Performance of stepped-septum polarizer with Teflon slab of Fig. 4 in place. (a) Axial ratio with port 1 excited and port 2 match loaded. Port 2 performance was similar. (b) Isolation. (c) VSWR of port 1 -right-hand circular polarization. (d) VSWR of port 2-lefthand circular polarization. VSWR is less than 1.2 for both (c) and (d) which are expanded 1.5 VSWR Smith chart plots.

formance of the polarizer is shown in Fig. 5. It is seen that while improving the axial ratio the slab did not adversely affect either the VSWR or the isolation.

\section{Comments}

The stepped-septum polarizer described in this communication gives significantly better performance over a wider bandwidth than the sloping-septum device.

In an actual phased-array application, because of lattice limitations, the square-waveguide radiators will almost certainly have to be dielectrically loaded for scan ranges of the order of $\frac{1}{4}$ hemisphere or greater [2]. In such cases all dimensions of the polarizer of Fig. 3 are to be scaled by the factor $1 /\left(\epsilon_{r}\right)^{1 / 2}$ where $\epsilon_{r}$ is the dielectric constant of the material filling the waveguide. The same scaling applies to the dimensions of the phase-correcting dielectric slab (Fig. 4) which will have to be of dielectric constant $\epsilon_{r}^{\prime}$ such that $\epsilon_{\tau}{ }^{\prime}=2.1 \epsilon_{r}$. This method of correcting phase may not be suitable if the device is used in high-power applications and is, in general, very cumbersome for dielectrically filled waveguides. A simpler method for both cases would be to introduce a small step in two opposite walls of the square waveguide following the septum in order to delay one mode relative to the other.

At first sight it might appear that the size of the square waveguide is too restrictive $\left(a=0.626 \lambda_{0} /\left(\epsilon_{r}\right)^{1 / 2}\right)$. This is a consequence of the fact that square waveguides are capable of only about 34 percent bandwidth, under the already stated assumption that all higher order modes are below cutoff. A 20 percent bandwidth capability thus leaves a margin of about 14 percent, part of which $(\sim 12$ percent) is used as guard between the lower edge of the operating band and the waveguide dominant-mode cutoff, for good matching, while the remainder ( $\sim 2$ percent) is the guard between the upper edge of the operating band and the cutoff of the first $\left(\mathrm{TE}_{11}\right)$ higher order mode. These margins may be juggled somewhat but probably not appreciably so that a restriction on waveguide size may be met by choosing an appropriate dielectric material. It is believed that a 25 percent bandwidth is close to the maximum practical operating bandwidth for a square-waveguide polarizer or radiator [2] free of higher order modes.

Although this polarizer may be used for any bandwidth less than 20 percent as it stands, a reoptimization procedure would probably yield better performance. For narrow bandwidths $(\sim 2-5$ percent) the sloping-septum polarizer [4] appears to be adequate.

\section{AckNowledgmext}

The authors acknowledge the effort of $\mathrm{L}$. Gerrior in assisting with the experimental program.

\section{REFERENCES}

[1] G, H. Knittel and G. N. Tsandoulas, "Square waveguide radiating elements for dual-polarization phased arrays-formulation and solution of the problem," in 1972 G-AP Symp. Dig. pp. 153-156.

[2] $G$. $N$. Tsandoulas and $G$. $H$. Knittel, "The design and performance Dig., pp. 157-160.

[3] A. J. Simmons, "Phase shift by periodic loading of waveguide and its application to broad-band circular polarization," IRE Trans. Microuate Theory Tech. rol. MTT-3, pp. 18-21, Dec. 1955.

[4] D. Davis, O J. Digiandomenico, and J. A. Kempic, "A new type of circularly polarized antenna element," in 1967 G-AP Symp. Dig., pp. 26-33.

[a] D. Frediani, private communication.

\section{On the Scattering Cross Section of Passive Linear Arrays}

\section{SOLYMAR}

Abstract-A general formula is derived for the scattering cross section of a passive $n$-element linear array consisting of isotropic radiators. When all the reactances are tuned out and scattering in the mirror direction is investigated, it is found that $A_{s r}$, the relative scattering cross section is equal to the square of the maximum gain the array can produce. As a consequence, for forward scattering in the limiting case of zero spacing between the elements, $A_{s \tau}=n^{4}$.

The cross section of a scattering object having cylindrical symmetry is defined as follows

$\mathrm{A}_{s}\left(\theta_{1}, \theta_{2}\right)$

$$
=4 \pi r^{2} \frac{\text { power density at distant point } r \text { in direction } \theta_{2}}{\text { power density of plane wave incident from direction } \theta_{1}} .
$$

For an array one is more interested in the relative scattering cross section

$$
A_{s r}\left(\theta_{1}, \theta_{2}\right)=\frac{A_{s}\left(\theta_{1}, \theta_{2}\right)}{A_{s i}\left(\theta_{1}, \theta_{2}\right)}
$$

where $A_{s i}$ is the scattering cross section of the individual radiator.

In this Communication we shall express $A_{s_{r}}\left(\theta_{3}, \theta_{2}\right)$ in terms of the geometry of the array and of the parameters of the matching matrix. A general formulation for an arbitrary three-dimensional array is certainly possible, but we shall, for simplicity, restrict the investigation to linear arrays consisting of isotropic radiators. In that particular case there are several theorems available making

Manuscript received December 4, 1972; revised January 11, 1973. The author is with the Laboratory of Electromagnetic Theory, the Technical University of Denmark, Lyngby, Denmark. 


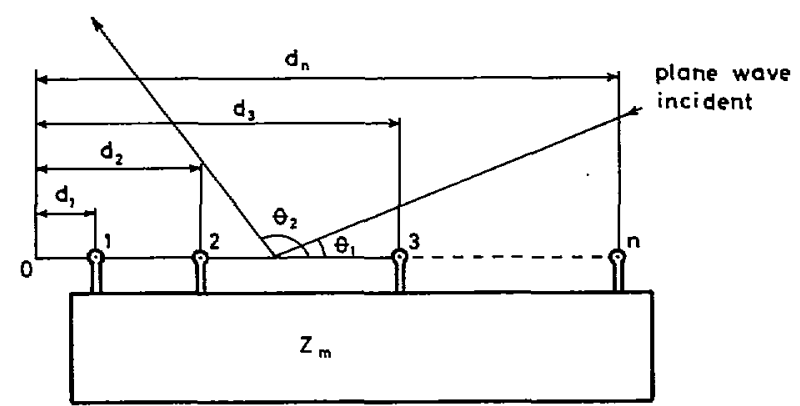

Fig. 1

it easier to evaluate the results. We shall also assume that the radiator considered has zero scattering cross-section when opencircuited so a single equivalent circuit may be used valid both for reception and reradiation.

Consider now the array of Fig. 1 where $Z_{m}$ is a linear passive matching network connected to the terminals of the array. The element positions are $d_{1}, d_{2}, \cdots, d_{n}$ measured from an arbitrarily chosen zero.

The voltage induced in a single element may be written in the form

$$
e=C_{1} S_{\mathbf{i}}{ }^{1 / 2}
$$

where $S_{\text {in }}$ is the incident power density and $C_{1}$ is a constant the value of which is of no interest in the present context. Taking the phase zero at the chosen zero point, the excitation of the $l$ th element is as follows

$$
e_{l}=C_{1} S_{\mathrm{in}}^{1 / 2} \exp j k d_{l} \cos \theta_{1}
$$

which can also be written as

$$
e=C_{1} S_{\mathrm{in}}^{1 / 2} f\left(\theta_{1}\right)
$$

where the $n$-dimensional vectors $e$ and $f$ are defined as

$$
e=\left[\begin{array}{c}
e_{1} \\
e_{2} \\
\vdots \\
e_{n}
\end{array}\right] \text { and } f(\theta)=\left[\begin{array}{c}
\exp j k d_{1} \cos \theta \\
\exp j k d_{2} \cos \theta \\
\vdots \\
\exp j k d_{n} \cos \theta
\end{array}\right]
$$

The current flowing in the elements will depend on the self- and mutual-impedances of the array and on the matching network but can always be expressed in the form

$$
i=\bar{Y} e
$$

where $\bar{Y}$ is an $n \times n$ admittance matrix and $i$ is an $n$-dimensional vector

$$
\boldsymbol{i}=\left[\begin{array}{c}
i_{1} \\
i_{2} \\
\vdots \\
i_{n}
\end{array}\right]
$$

The power density produced at the distance $r$ by a single radiator of current $i_{l}$ is

$$
S(r)=C_{2} \frac{i t^{2}}{4 \pi r^{2}}
$$

where $C_{2}$ is another constant. The power density produced by the array is

$$
S(r)=\frac{C_{2}}{4 \pi r^{2}}\left|f\left(\theta_{2}\right) i\right|^{2}
$$

Substituting for $i$ from (7) and using (5) we get

$$
S(r)=\frac{C_{1}^{2} C_{2} S_{\text {in }}}{4 \pi r^{2}}\left|f\left(\theta_{2}\right) \boldsymbol{Y} \boldsymbol{f}\left(\theta_{1}\right)\right|^{2}
$$

Hence the relative scattering eross section (2) is of the form

$$
A_{s r}=R_{11}^{2}\left|f\left(\theta_{2}\right) \bar{Y} f\left(\theta_{1}\right)\right|^{2}
$$

where $R_{11}$ is the self-resistance of the individual elements and the self-reactance is assumed to be zero. Note that $A_{s T}$ equals unity for $n=1$.

Let us now investigate the special case when the matching network is chosen so as to tune out all the mutual reactances that is

$$
\bar{Y}=\bar{R}^{-1}
$$

where $R$ is the real part of the impedance matrix of the array. Choosing further

$$
\theta_{2}=\pi-\theta_{1}
$$

that is evaluating the scattering in the mirror direction, we get

$$
A_{s}=\left[f^{*}\left(\theta_{1}\right) R_{11} R^{-1} f\left(\theta_{1}\right)\right]^{2} .
$$

The expression in the bracket may be recognized to be the maximum gain an array can produce in the $\theta_{1}$ (or $\pi-\theta_{1}$ ) direction. Thus

$$
A_{s r}\left(\theta_{1}, \pi-\theta_{1}\right)=G_{\max }{ }^{2}\left(\theta_{1}\right) \text {. }
$$

An interesting limiting case is $\theta_{1}=0$ when the distance between the elements tends to zero. Then, according to Uzkov [1] the maximum gain is equal to $n^{2}$ leading to

$$
A_{s r}(0, \pi)=n^{4}
$$

Equations (16) and (17) prove not only that linear arrays may have "supergain" qualities as scatterers but also that they can be "supergain" as "receivers" from the $\theta_{1}$ direction and reradiators in the $\theta_{2}$ direction at the same time. It is easy to account for the latter property. A plane wave incident from the $\theta_{1}$ direction will induce equal voltage amplitudes and just the right phase for radiating in the $\pi-\theta_{1}$ direction. According to the travelling wave theorem of Bloch et al. [2] this voltage distribution will give the maximum gain.

It is more difficult to devise a simple argument for explaining the "receiving" properties of the array but perhaps it is only due to our unfamiliarity with the subject. One usually looks at transmission and reception separately and perhaps when they occur simultaneously there is little benefit in looking at them separately. A separation though is possible. One can express the power going into the array (and necessarily reradiated if all the matching elements are reactive) as follows

$$
\begin{aligned}
P & =\frac{1}{2} \operatorname{Re} e^{*} i \\
& =\frac{1}{2} \operatorname{Re} e^{* \bar{Y}} e \\
& =\frac{1}{2} C_{1}^{2} S_{\mathrm{in}_{\mathrm{B}}} \operatorname{Re} f^{*}\left(\theta_{1}\right) \bar{Y} f\left(\theta_{1}\right) .
\end{aligned}
$$

Choosing now $\bar{Y}$ in the form of (13) it may be seen that $P$ becomes proportional to $G_{\mathrm{max}}\left(\theta_{1}\right)$. The gain in the mirror direction is also $G_{\max }\left(\theta_{1}\right)$; so according to this alternative derivation of (16) both "reception" and reradiation are proportional to $G_{\max }$. Note, however, that this relationship is no longer valid when scattering in other than mirror direction is considered.

\section{ACKNOWLEDGMENT}

The author wishes to thank Dr. techn. J. Bach Andersen for a critical reading of the manuscript and for a number of valuable discussions.

\section{REFERENCES}

[1] A. I. Uzkov, "An approach to the problem of optimum directive antenna design," C. R. Acad. Sci. USSR, voli 53, pp. 35-38, 1946. 2] A. Bloch, R. G, Medhurst, and S. D. Pool, "A new approach to the design of superdirective aerial
vol. 100, pp. $303-314,1953$. 